\title{
A Procedure for Conformal Maps of Simply Connected Domains by Using the Bergman Function
}

\author{
By J. Burbea*
}

\begin{abstract}
Conformal maps of simply connected domains onto the unit circle are computed by means of the Bergman function of the domain. Ellipses and squares are mapped by this method. Further various parameters of the Schwarz-Christoffel formula are computed in terms of the Bergman function.
\end{abstract}

1. Introduction. Conformal maps of simply connected domains onto the unit circle are computed by means of the Bergman kernel function of the domain. As an example of this method we map ellipses and squares and compare numerical results to known results. We also compute various parameters of the Schwarz-Christoffel formula in terms of the kernel function. The computation is programmed in Fortran and run on the Golem-1 and CDC 1604 at the Weizmann Institute of Science, Rehovot, Israel. In general the method gives good accuracy. For twofour- and eightfold symmetric domains the method can be simplified to decrease the amount of computation needed to achieve the same accuracy as would be achieved by the method for the general case.

2. General Theory. Let $D$ be a bounded simply connected domain in the $z$-plane with the boundary $C$. Consider the separable Hilbert space $\mathcal{L}^{2} H(D)$, which consists of all square integrable analytic functions in $D$. Let $\left\{\varphi_{\nu}(z)\right\}_{\nu=1}^{\infty}$ be any orthonormal basis of $\mathscr{L}^{2} H(D)$. As is well known, the Bergman kernel function

$$
K_{D}(z, \bar{t})=\sum_{\nu=1}^{\infty} \varphi_{\nu}(z) \overline{\varphi_{\nu}(t)}
$$

is independent of the choice of the orthonormal basis and belongs to $\mathscr{L}^{2} H(D)$ as a function of $z$ or $\bar{t}$. The kernel function is also uniquely characterized by its reproducing property, namely, for each $f \in \mathscr{L}^{2} H(D)$ we have

$$
f(t)=\iint_{D} f(z) \overline{K(z, \bar{t})} d x d y=(f, K(z, t)),
$$

where $(f, g)$ denotes the scalar product in $\mathfrak{L}^{2} H(D)$, i.e., for $f, g \in \mathscr{L}^{2} H(D)$ we have

$$
(f, g)=\iint_{D} f(z) \overline{g(z)} d x d y .
$$

Received February 24, 1970.

AMS 1969 subject classifications. Primary 6510, 6520.

Key words and phrases. Bergman kernel function, conformal maps, reproducing property, Gaussian quadrature rule.

* This work was supported in part by Contracts AEC 326-P22 and AFOSR F44620-69-C-0106 at Stanford University.

The author wishes to thank Professor P. Rabinowitz, Weizmann Institute of Science, for his valuable help. 
Here we have

$$
\left(\varphi_{\nu}, \varphi_{\mu}\right)=\delta_{\nu \mu} .
$$

The kernel function is relative invariant, that is, if $w=f(z)$ maps $D$ conformally onto $D^{*}$, then

$$
K_{D}(z, \bar{t})=K_{D^{*}}(w, \bar{v}) f^{\prime}(z) \overline{f^{\prime}(t)}, \quad v=f(t) .
$$

If $D^{*}$ is the unit circle, then the mapping function $w=f(z)$. which satisfies

$$
f(t)=0, \quad f^{\prime}(t)>0 .
$$

where $t$ is a given point in $D$, is given by

$$
f(z)=\left(\frac{\pi}{K(t, \bar{t})}\right)^{1 / 2} \int_{t}^{z} K(\xi, \bar{t}) d \xi, \quad K(\xi, \bar{t})=K_{D}(\xi, \bar{t}) .
$$

As is well known, if the complement of $D$ is a closed domain, then the set $\left\{z^{i}\right\}_{i=0}^{\infty}$ forms a complete set in $\mathscr{L}^{2} H(D)$. By applying the Gram-Schmidt procedure, we obtain the sequence of the orthonormal polynomials $\left\{P_{n}(z)\right\}_{n=0}^{\infty}$ given by

$$
P_{n}(z)=\sum_{k=0}^{n} a_{n k} z^{k}, \quad n=0,1,2, \cdots .
$$

The $\left\{a_{n k}\right\}$ are determined recursively by

$$
a_{n k}=c_{n k} /\left(\sum_{i=0}^{n} \sum_{i=0}^{n} c_{n i} \bar{c}_{n i}\left(z^{i}, z^{i}\right)\right)^{1 / 2},
$$

where

$$
c_{n n}=1 ; \quad c_{n k}=-\sum_{i=k}^{n-1} a_{i k}\left(\sum_{i=0}^{i} \bar{a}_{i j}\left(z^{n}, z^{j}\right)\right), \quad k=0,1,2, \cdots, n-1 .
$$

The computational procedure is as follows. The domain $D$ is given by its boundary $C$ which is described by a set of $N$ complex numbers $z_{k}=x_{k}+i y_{k}, k=1,2, \cdots, N$. Using these numbers, we determine the values of the functions $1, z, z^{2}, \cdots, z^{n}$ along the boundary. They are represented by the $N$-dimensional complex valued vector of its values at the points $z_{k}, k=1, \cdots, N$.

We use the Green formula to evaluate the moments

$$
\left(z^{m}, z^{n}\right)=\iint_{D} z^{m_{z^{n}}} d x d y=\frac{1}{2 i(n+1)} \int_{C} z^{m_{z^{n+1}}} d z .
$$

Numerically the moments are approximated by the Gaussian quadrature rule (48 points) (see [3]), that is,

$$
\left(z^{m}, z^{n}\right) \cong \frac{1}{2 i(n+1)} \sum_{k=1}^{N} w_{k} z_{k}^{m} z_{k}^{n+1}
$$

Here, $w_{k}$ are the positive weights as given in [3] for 48 points. Then using (2.9) and (2.10), we obtain $\left\{P_{k}(z)\right\}_{k=0}^{n}$, see (2.8), whence, after determining the point $t$, we form the sum

$$
K_{n}(z, \bar{t})=\sum_{k=0}^{n} P_{k}(z) \overline{P_{k}(t)} .
$$


After formal integration of the polynomials $\left\{P_{k}(z)\right\}_{k=0}^{n}$, we obtain the coefficients of the approximated mapping function $f_{n}(z)$. The quality of the approximation and the rate of convergence can be determined by the evaluation of $f_{n}(z)$ at the points $\left\{z_{k}\right\}_{k=1}^{N}$ to see how close they are to the unit circle.

Following Rabinowitz [6], the notation used is as follows. $N$ is the number of points on the boundary of $D ; n$ is the number of functions used, and we define

$$
\begin{aligned}
& \max \delta\left(f_{n}\right)=\max _{k=1, \cdots, N}\left|1-f_{n}\left(z_{k}\right)\right|, \\
& \min \delta\left(f_{n}\right)=\min _{k=1, \cdots, N}\left|1-f_{n}\left(z_{k}\right)\right| .
\end{aligned}
$$

We write $\infty$ for values greater than $10^{4}, n_{\max }$ is the value of $n$ up to which orthonormal polynomials $\left\{P_{k}(z)\right\}_{k=0}^{n}$ were computed. $n_{\min }$ is the smallest $n$ for which $f_{n}$ is computed. $n_{\text {opt }}$ is that value of $n$ among $n_{\min }$ and $n_{\max }$ for which $f_{n}$ was computed and for which $\max \delta$ was a minimum $\left(n_{\min } \leqq n_{\mathrm{opt}} \leqq n_{\max }\right)$.

\section{Normalized Ellipses.}

Example 1. Let $D$ be the ellipse

$$
D=\left\{\langle x, y\rangle: b^{2} x^{2}+a^{2} y^{2} \leqq a^{2} b^{2}\right\}, \quad z=x+i y,
$$

where

$$
a^{2}-b^{2}=1,
$$

i.e., the foci of $D$ are located at $z= \pm 1$. As is known (see [5, p. 260]), the mapping function $f(z)$ which maps $D$ onto the unit circle such that

$$
f(0)=0, \quad f^{\prime}(0)>0
$$

is given by

$$
\begin{gathered}
f(z)=2 \alpha \sum_{n=0}^{\infty} \frac{(-1)^{n} \cos (2 n+1) w}{\rho^{2 n+1}-\rho^{-2 n-1}}, \\
\alpha^{-2}=\sum_{n=0}^{\infty} \frac{2 n+1}{\rho^{2 n+1}-\rho^{-2 n-1}}, \quad \rho=(a+b)^{2}, \\
w=\cos ^{-1} z .
\end{gathered}
$$

In the first quadrant of the ellipse we have

$$
w=\cos ^{-1} z=-i \log \left(z+\left(z^{2}-1\right)^{1 / 2}\right) .
$$

We choose $a=2(0.2) 3$ and compare the results obtained by using (3.3)-(3.7) with those obtained by the method described in Section 2. Here,

$$
\begin{array}{ll}
z_{k}=x_{k}+i y_{k}, \quad x_{k}=a \cos \theta_{k}, \quad y_{k}=b \sin \theta_{k}, \quad \theta_{k}=(k-1) \pi / 90, \\
w_{k}=u_{k}+i v_{k}=\operatorname{Re}\left\{f\left(z_{k}\right)\right\}+i \operatorname{Im}\left\{f\left(z_{k}\right)\right\}=f\left(z_{k}\right), \quad k=1,2, \cdots, 46 .
\end{array}
$$

The number of terms in the sums (3.4) and (3.5) will be determined by the criterion that the ratio of the additional term over the new sum is less than $\epsilon=10^{-12}$. Table 1 describes the results obtained due to (3.3)-(3.7) for $a=2.6$ and $b=2.4$, and Table 2 describes the results obtained from the method described in Section 2 for the same 
TABLE 1

\begin{tabular}{llllll}
\hline$\theta_{k}$ & \multicolumn{1}{c}{$x_{k}$} & \multicolumn{1}{c}{$y_{k}$} & \multicolumn{1}{c}{$u_{k}$} & \multicolumn{1}{c}{$v_{k}$} & $\left|f\left(z_{k}\right)\right|$ \\
\hline$\theta_{1}$ & 2.600 & .000 & 1.000 & $2.359 \cdot 10^{-12}$ & 1.000 \\
$\theta_{5}$ & 2.575 & $3.340 \cdot 10^{-1}$ & $9.930 \cdot 10^{-1}$ & $1.182 \cdot 10^{-1}$ & 1.000 \\
$\theta_{16}$ & 2.252 & 1.200 & $8.979 \cdot 10^{-1}$ & $4.402 \cdot 10^{-1}$ & 1.000 \\
$\theta_{20}$ & 2.049 & 1.478 & $8.329 \cdot 10^{-1}$ & $5.535 \cdot 10^{-1}$ & 1.000 \\
$\theta_{31}$ & 1.300 & 2.078 & $5.598 \cdot 10^{-1}$ & $8.286 \cdot 10^{-1}$ & 1.000 \\
$\theta_{46}$ & $3.450 \cdot 10^{-6}$ & 2.400 & $1.548 \cdot 10^{-1}$ & 1.000 & 1.000 \\
\hline
\end{tabular}

$a$ and $b$ but $n \geqq 7$. Note the fast convergence of this mapping. This is mostly due to the fact that $a \gg 1$, here, $a=2.6$ and hence, $b=\left(a^{2}-1\right)^{1 / 2}=2.4$; thus, $b$ is of the same order of magnitude of $a$. Therefore, the ellipses are in this case close to circles. Thus, the Gram determinant is far away from zero and the powers of $z$ are almost orthogonal. We will return to the ellipses in the next section.

4. Fourfold Symmetric Domains. Let $D$ be a simply connected fourfold symmetric domain around the origin. Let us seek the mapping function which maps $D$ onto the unit circle such that the origin is mapped onto the origin. Here, the boundary $C$ is composed of four symmetrical parts

$$
C=\bigcup_{k=1}^{4} C_{k}
$$

The moments $\left(z^{m}, z^{n}\right)$ are given by

$$
\left(z^{m}, z^{n}\right)=\left\{\begin{array}{lll}
0, & m-n \neq \equiv 0 & (\bmod 2), \\
\frac{2}{n+1} \int_{C_{1}}|z|^{2 m} \operatorname{Im}\left[\bar{z}^{n-m+1} d z\right], & m-n \equiv 0 \quad(\bmod 2) .
\end{array}\right.
$$

The orthonormal polynomials $\left\{P_{n}(z)\right\}_{n=0}^{\infty}$ are given as in (2.8)-(2.10); however,

$$
\begin{aligned}
& a_{m n}=\bar{a}_{m n} ; \quad c_{m n}=\bar{c}_{m n}, \quad n \leqq m, \\
& a_{m n}=c_{m n}=0, \quad m-n \not \equiv 0 \quad(\bmod 2) .
\end{aligned}
$$

TABLE 2

\begin{tabular}{llllll}
\hline$\theta_{k}$ & \multicolumn{1}{c}{$x_{k}$} & \multicolumn{1}{c}{$y_{k}$} & \multicolumn{1}{c}{$u_{k}$} & \multicolumn{1}{c}{$v_{k}$} & $\left|f\left(z_{k}\right)\right|$ \\
\hline$\theta_{1}$ & 2.600 & .000 & 1.000 & .000 & 1.000 \\
$\theta_{5}$ & 2.575 & $3.340 \cdot 10^{-1}$ & $9.930 \cdot 10^{-1}$ & $1.181 \cdot 10^{-1}$ & 1.000 \\
$\theta_{16}$ & 2.252 & 1.200 & $8.979 \cdot 10^{-1}$ & $4.402 \cdot 10^{-1}$ & 1.000 \\
$\theta_{20}$ & 2.049 & 1.478 & $8.329 \cdot 10^{-1}$ & $5.535 \cdot 10^{-1}$ & 1.000 \\
$\theta_{31}$ & 1.300 & 2.078 & $5.598 \cdot 10^{-1}$ & $8.286 \cdot 10^{-1}$ & 1.000 \\
$\theta_{46}$ & $3.450 \cdot 10^{-6}$ & 2.400 & $1.548 \cdot 10^{-6}$ & 1.000 & 1.000 \\
\hline
\end{tabular}


Therefore, $\overline{P_{n}(z)}=P_{n}(\bar{z})$ and further

$$
P_{2 m}(z)=\sum_{k=0}^{m} a_{2 m, 2 k} z^{2 k} ; \quad P_{2 m+1}(z)=\sum_{k=0}^{m} a_{2 m+1,2 k+1} z^{2 k+1} .
$$

Here,

$$
K(z, 0)=\sum_{m=0}^{\infty} P_{2 m}(z) P_{2 m}(0)=\sum_{m=0}^{\infty} a_{2 m, 0} P_{2 m}(z) .
$$

Let $w=f(z)$ be the mapping function from $D$ onto the unit circle such that

$$
f(0)=0, \quad f^{\prime}(0)>0 .
$$

Then

$$
f(z)=S \sum_{n=0}^{\infty} \frac{z^{2 n+1}}{2 n+1}\left(\sum_{k=n}^{\infty} a_{2 k, 0} a_{2 k, 2 n}\right)
$$

where

$$
S=\frac{\pi}{\sum_{n=0}^{\infty} a_{2 n, 0}^{2}} .
$$

We have the following significant simplification. First the odd powers of $z$ do not appear in the kernel function, as is seen in (4.2). Therefore, only orthonormalization of the even powers of $z$ is needed. Further the nonvanishing moments are given by

$$
\left(z^{m}, z^{n}\right)=\frac{2}{n+1} \int_{C_{1}} \operatorname{Im}\left[z^{m_{\bar{z}} n+1}\right] d x+\frac{2}{n+1} \int_{C_{1}} \operatorname{Re}\left[z^{m_{z^{n+1}}^{n}}\right] d y .
$$

Numerically we use the trapezoidal rule or the Gaussian quadrature rule, but instead of $N$ we actually take

$$
N_{1}=N / 4+1 .
$$

Example 2. For the sake of comparison, see [6, Example 2, Table 3], we let $D$ be the ellipse

$$
\begin{aligned}
D & =\left\{\langle x, y\rangle: \frac{x^{2}}{a^{2}}+y^{2}<1\right\}, \\
N=180, \quad \theta_{k} & =(k-1) \pi / 90, \quad k=1,2, \cdots, N_{1} .
\end{aligned}
$$

TABLE 3

\begin{tabular}{cclcccll}
\hline$a$ & $n_{\min }$ & $\max \delta$ & $n_{\max }$ & $\max \delta$ & $n_{\mathrm{opt}}$ & $\max \delta$ & $\min \delta$ \\
\hline 1.25 & 7 & .000002 & 19 & .000004 & 7 & .000002 & .000000 \\
2.50 & 4 & .023 & 19 & 620 & 10 & .000061 & .000000 \\
5.00 & 4 & .16 & 13 & 159 & 9 & .015 & .0005 \\
10.0 & 4 & .41 & 13 & $\infty$ & 7 & .20 & .01 \\
20.00 & 4 & .65 & 13 & $\infty$ & 7 & .45 & .003 \\
\hline
\end{tabular}


Table 3 describes the results of Rabinowitz, while Table 4 describes the results of our method. In these tables max $\delta$ is computed for $n_{\min }, n_{\max }$ and $n_{\text {opt }}$, respectively. For large $a$ the roundoff error is very large in the case of Table 3. The influence of the roundoff on the mapping function for large $a$ in the Rabinowitz case is explained in [6] as follows. For large $a$ the ellipses are so thin as to have the properties of line segments and that orthonormalizing the power of $z$ on a line segment is equivalent to inverting a Hilbert matrix which is nearly singular.

Integration in the case of Rabinowitz is over the boundary of the domain, and hence, he exploits the Szegö kernel function. However, as is seen from Table 4, we do not have in our method this ill-conditioned problem. This is due to the fact that in our case integration is over the domain $D$, and hence, though the ellipses are very thin, they still possess a finite area which ensures the Gram determinant to be far away from zero. Therefore, adding of functions to the system of functions needed for the orthonormalization (up to a certain number of functions) improves the approximation and does not spoil it. For $a=1.25,2.50$ it should be understood that $n \geqq n_{\mathrm{opt}}$ gives the same result in Table 4 . In general, we have $n_{\mathrm{opt}} \cong n_{\max }$, even for $a=20$ and $n=24$ we still have $\max \delta=.611$. However, $n_{\mathrm{opt}}$ between 4 and 13 is not 13 but $n_{\mathrm{opt}}=11$. After this number the roundoff error is larger. The reason for this is that for large $n, z_{k}^{n}=\left(a \cos \theta_{k}+i b \sin \theta_{k}\right)^{n}, a \gg b$, is almost equal to $z_{k}^{n-1}$. Hence, the Gram determinant is very near to zero, which spoils the orthonormalization. For $a=30$ the roundoff error is very bad, as is seen from Table 5. Here $\theta_{k}$, $x_{k}, y_{k}, u_{k}, v_{k},\left|f\left(z_{k}\right)\right|$ are given as in (3.8), (3.9), but $a=30, b=1$ and $n=18$. Table 5 is computed according to the method described in Section 2.

5. Eightfold Symmetric Domains. For domains with eightfold symmetry around the origin we obtain twice the simplification obtained for fourfold symmetric domains around the origin. Analogously to Section 4 we have

$$
C=\bigcup_{k=1}^{8} C_{k} .
$$

The moments are given via

$$
\left(z^{m}, z^{n}\right)=\left\{\begin{array}{lll}
0, & m-n \neq 0 \quad(\bmod 4), \\
\frac{4}{n+1} \int_{C_{1}}|z|^{2 m} \operatorname{Im}\left[\bar{z}^{n-m+1} d z\right], & m-n \equiv 0 \quad(\bmod 4) .
\end{array}\right.
$$

The orthonormal polynomials are given by

TABLE 4

\begin{tabular}{rccccccc}
\hline \multicolumn{1}{c}{$a$} & $n_{\min }$ & $\max \delta$ & $n_{\max }$ & $\max \delta$ & $n_{\mathrm{opt}}$ & $\max \delta$ & $\min \delta$ \\
\hline 1.25 & 4 & .0002 & 19 & .0000 & 5 & .0000 & .0000 \\
2.50 & 4 & .0385 & 19 & .0000 & 11 & .0000 & .0000 \\
5.00 & 4 & .1950 & 13 & .0122 & 13 & .0122 & .0010 \\
10.00 & 4 & .5360 & 13 & .0964 & 13 & .0964 & .0012 \\
20.00 & 4 & 1.1040 & 13 & .2920 & 11 & .2240 & .0068 \\
\hline
\end{tabular}


TABle 5

\begin{tabular}{llllll}
\hline$\theta_{k}$ & \multicolumn{1}{c}{$x_{k}$} & \multicolumn{1}{c}{$y_{k}$} & \multicolumn{1}{c}{$u_{k}$} & \multicolumn{1}{c}{$v_{k}$} & \multicolumn{1}{c}{$\left|f\left(z_{k}\right)\right|$} \\
\hline$\theta_{1}$ & 30.00 & .000 & 1.391 & .000 & 1.391 \\
$\theta_{5}$ & 29.71 & $1.392 \cdot 10^{-1}$ & $6.902 \cdot 10^{-1}$ & $7.565 \cdot 10^{-2}$ & $6.943 \cdot 10^{-1}$ \\
$\theta_{16}$ & 25.98 & $.500 \cdot 10^{-1}$ & $8.821 \cdot 10^{-1}$ & $-2.523 \cdot 10^{-1}$ & $9.175 \cdot 10^{-1}$ \\
$\theta_{20}$ & 23.64 & $6.157 \cdot 10^{-1}$ & 1.288 & $2.465 \cdot 10^{-1}$ & 1.312 \\
$\theta_{31}$ & 15.00 & $8.660 \cdot 10^{-1}$ & $4.933 \cdot 10^{-1}$ & $7.059 \cdot 10^{-2}$ & $4.983 \cdot 10^{-1}$ \\
$\theta_{46}$ & $3.98 \cdot 10^{-5}$ & 1.000 & $3.521 \cdot 10^{-5}$ & $8.007 \cdot 10^{-1}$ & $8.007 \cdot 10^{-1}$ \\
\hline
\end{tabular}

$$
P_{4 m+l}(z)=\sum_{k=0}^{m} a_{4 m+l, 4 k+l} z^{4 k+l}, \quad l=0,1,2,3 .
$$

Further,

$$
K(z, 0)=\sum_{m=0}^{\infty} a_{4 m, 0} P_{4 m}(z) .
$$

The mapping function from the eightfold symmetric domain $D$ onto the unit circle satisfying (4.7) is given by

$$
f(z)=S \sum_{n=0}^{\infty} \frac{z^{4 n+1}}{n+1}\left(\sum_{k=n}^{\infty} a_{4 k, 0} a_{4 k, 4 n}\right),
$$

where

$$
S=\frac{\pi}{\sum_{n=0}^{\infty} a_{4 n, 0}^{2}} .
$$

Numerically, we use the Gaussian quadrature rule for 48 points, where we actually need

$$
N_{1}=N / 8+1 \text {. }
$$

The only powers of $z$ which appear in the kernel function are those which are multiples of 4. Hence, we only orthonormalize those powers of $z$.

Example 3. Let $D$ be the square with the boundary

$$
C=\{\langle x, y\rangle:|x|=a,|y| \leqq a \text { or }|y|=a,|x| \leqq a\} .
$$

Here,

$$
C_{1}=\{\langle x, y\rangle: x=a, 0 \leqq y \leqq a\} .
$$

The moments are given by (for $m-n \equiv 0(\bmod 4)$ )

$$
\left(z^{m}, z^{n}\right)=\frac{4}{n+1} a^{m+n+2} \int_{0}^{1}\left(x^{2}+1\right)^{m} \operatorname{Im}\left[(x+i)^{n-m+1}\right] d x .
$$

The first five orthonormal polynomials are given by

$$
\begin{aligned}
& P_{0}(z)=1 / 2 a ; \quad P_{1}(z)=\left(1 / 2 a^{2} \cdot(3 / 2)^{1 / 2}\right) z ; \quad P_{2}(z)=\left(3 / 4 a^{3}(5 / 7)^{1 / 2}\right) z^{2} \\
& P_{3}(z)=\left(1 / a^{4}(35 / 96)^{1 / 2}\right) z^{3} ; \quad P_{5}(z)=\left(1 / a^{5}(7 / 19456)^{1 / 2}\right)\left(30 z^{4}+8 a^{4}\right) .
\end{aligned}
$$


TABLE 6

\begin{tabular}{rccccl}
\hline$k$ & $x_{k}$ & \multicolumn{1}{c}{$y_{k}$} & \multicolumn{1}{c}{$u_{k}$} & \multicolumn{1}{c}{$v_{k}$} & \multicolumn{1}{c}{$\left|f\left(z_{k}\right)\right|$} \\
\hline 1 & .7 & .0 & $9.847 \cdot 10^{-1}$ & .0 & $9.847 \cdot 10^{-1}$ \\
6 & .7 & $1.750 \cdot 10^{-1}$ & $9.493 \cdot 10^{-1}$ & $2.957 \cdot 10^{-1}$ & $9.942 \cdot 10^{-1}$ \\
11 & .7 & $3.500 \cdot 10^{-1}$ & $8.566 \cdot 10^{-1}$ & $5.381 \cdot 10^{-1}$ & 1.012 \\
16 & .7 & $5.250 \cdot 10^{-1}$ & $7.479 \cdot 10^{-1}$ & $6.810 \cdot 10^{-1}$ & 1.012 \\
21 & .7 & $7.000 \cdot 10^{-1}$ & $6.918 \cdot 10^{-1}$ & $6.918 \cdot 10^{-1}$ & $9.784 \cdot 10^{-1}$ \\
\hline
\end{tabular}

Numerically, we take

$$
x_{k}=a ; \quad y_{k}=(k-1) \frac{a}{20} ; \quad k=1,2, \cdots, 21 .
$$

Table 6 describes the results for $a=.7, n=5$ for 48 points of the Gaussian quadrature rule. Note that the results of Table 6 could also be obtained if integration was done analytically as in (5.11) since the 48 points Gaussian quadrature is an exact integration for each polynomial of degree $\leqq 2.48-1=95$. As is shown in Table 6 , the corners of the square give poor results.

Example 4. Let $D$ be the square with the boundary

$$
C=\{\langle x, y\rangle:|x|=1,|y| \leqq 1 \text { or }|y|=1,|x| \leqq 1\} .
$$

By the Schwarz-Christoffel formula the mapping function of $D$ onto the unit circle is given by

$$
f(z)=\alpha z+\frac{\alpha^{5}}{10} z^{5}+\frac{\alpha^{9}}{120} z^{9}+\frac{11 \alpha^{3}}{15600} z^{13}+\cdots,
$$

where

$$
\alpha=\int_{0}^{1} \frac{d t}{\left(1+t^{4}\right)^{1 / 2}}=\frac{1}{2} \lll\left(\frac{1}{\sqrt{ } 2}\right)=.92703734
$$

(see [4, p. 159]). From (2.7) and (5.6) we also have

TABLE 7

\begin{tabular}{cccc}
\hline Term & Exact Value & $n=4$ & $n=7$ \\
\hline$\alpha$ & $9.270373386507 \cdot 10^{-1}$ & $9.270373343435 \cdot 10^{-1}$ & $9.270373386522 \cdot 10^{-1}$ \\
$\frac{\alpha^{3}}{10}$ & $6.846776221877 \cdot 10^{-2}$ & $6.846682430405 \cdot 10^{-2}$ & $6.846776221888 \cdot 10^{-2}$ \\
$\frac{\alpha^{9}}{120}$ & $4.213992852820 \cdot 10^{-3}$ & $4.207708065293 \cdot 10^{-3}$ & $4.213992852827 \cdot 10^{-3}$ \\
$\frac{11 \alpha^{13}}{15600}$ & $2.633492188752 \cdot 10^{-4}$ & $2.53460527343 \cdot 10^{-4}$ & $2.633492188653 \cdot 10^{-4}$ \\
\hline
\end{tabular}




$$
\alpha=f^{\prime}(0)=S K(0,0)=\left(\pi \sum_{n=0}^{\infty} a_{4 n, 0}^{2}\right)^{1 / 2} .
$$

Further, comparing (5.5) with (5.14) we obtain relations expressing the powers of $\alpha$ in terms of the coefficients which appear in the algorithm of the orthogonalization in (2.8)-(2.10). As an example we have

$$
\frac{\alpha^{5}}{10}=S \frac{1}{2} \sum_{k=1}^{\infty} a_{4 k, 0} a_{4 k, 4}
$$

and so on. We compute these coefficients by the method of the Bergman kernel function. For the orthonormalization we start with four functions and end with eleven functions. In Table 7 we compare the computed results of our method with the known values. It should be noted that from $n \geqq 7$ we have similar results for $n=11$.

Mathematics Department

Stanford University

Stanford, California 94305

1. S. Bergman, The Kernel Function and Conformal Mapping, Math. Surveys, no. 5, Amer. Math. Soc., Providence, R. I., 1950. MR 12, 402.

2. S. Bergman \& B. Chalmers, "A procedure of conformal mapping of triply-connected domains," Math. Comp., v. 21, 1967, pp. 527-542. MR 37 \#4243a.

3. P. DAvis \& P. RABINOWITz, "Abscissas and weights for Gaussian quadratures of high order," J. Res. Nat. Bur. Standards Sect. B, v. 56, 1965, pp. 35-37.

4. D. GaIER, Konstruktice Methoden der konformen Abbildung, Springer Tracts in Natural Philosophy, vol. 3, Springer-Verlag, Berlin, 1964. MR 33 \#7507.

5. Z. Nehari, Conformal Mapping, McGraw-Hill, New York, 1952. MR 13, 640.

6. P. RABINowITZ, "Numerical experiments in conformal mapping by the method of orthonormal polynomials," J. Assoc. Comput. Mach., v. 13, 1966, pp. 296-303. 